\title{
Screening results of high-risk population of elderly cerebral stroke in a community in Pudong New Area, Shanghai
}

Yumei Yang

East Hospital, Tongji University School of Medicine

Lingyu Li

East Hospital, Tongji University School of Medicine

Lei Feng

Sanlin Community Health Service Center

Xi Yang

Dongming Community Health Service Center

Feifeng Liu

East Hospital, Tongji University School of Medicine

Jinqiu Yi

Sanlin Kangde Community Health Service Center

Hui Dai

Sanlin Community Health Service Center

Zheng Liu

Dongming Community Health Service Center

bei zhang ( $\sim$ zhangbei0227@163.com )

East hospital https://orcid.org/0000-0002-5486-0941

\section{Research article}

Keywords: risk factors, cerebral stroke, prevention strategies, elderly

Posted Date: March 9th, 2020

DOI: https://doi.org/10.21203/rs.3.rs-16406/v1

License: (c) This work is licensed under a Creative Commons Attribution 4.0 International License. Read Full License 


\section{Abstract}

Objective To investigate the exposure of risk factors and the characteristics of population distribution of elderly patients with high-risk of cerebral stroke in Sanlin Community, Pudong New Area, Shanghai, aiming to provide the evidence for the development of the prevention strategies for elderly patients with high-risk of stroke.

Methods From 2015 to 2017, a cluster sampling method was adopted to conduct on-the-spot investigation and related physical examinations for resident populations $\geq 65$ years old in Sanlin Community of Pudong New Area. The demographic characteristics, medical history, blood lipids, fasting glucose, glycosylated hemoglobin, homocysteine and electrocardiogram were collected to screen the relative risk factors of ischemic stroke.

Results 9,195 residents were surveyed between 2015 and 2017. 1,504 residents were high-risk population and 535 residents $(5.82 \%)$ had a history of transient ischemic attack or stroke. There were 969 residents $(10.54 \%)$ with $\geq 3$ risk factors. Risk factors were hypertension, overweight, hyperlipidemia, diabetes mellitus, stroke history, smoking history, family history, lack of exercise and atrial fibrillation in turn. Among the high-risk groups of 969 risk factors $\geq$ 3 , women's diabetes and hyperlipidemia exposure rate was higher than that of men, and male smoking rate and overweight were higher than females. Among the community members who participated in the screening, the detection rate of hyperhomocysteinemia for male was $63.8 \%$, which was significantly higher than $45.7 \%$ for female.

Conclusion The detection rate of high-risk groups in people over 65 years old in Sanlin area is similar to that in other areas in Shanghai. Hypertension, overweight and hyperlipidemia are the highest exposure risk factors. There are differences in exposure to certain risk factors between men and women. There is a high detection rate of hyperhomocysteinemia in the elderly and male population. Regular follow-up management should be implemented in combination with the occurrence of risk factors in different populations in order to prevent stroke.

\section{Introduction}

Stroke is a severe disease that endangers human health with high incidence, high disability rate, high mortality and recurrence rate. Stroke has become the second most common cause of death and the first cause of disability in the world [1]. The risk of stroke in high-risk population is 10 times higher than that in non-high-risk population [2]. Screening the high-risk population of stroke and carrying out targeted interventions can effectively reduce the overall incidence and hazards of stroke. Age is also an important risk factor for stroke, and high-risk groups in the elderly are the key targets for stroke prevention and control. As a stroke screening base, Shanghai East Hospital affiliated to Tongji University, in conjunction with the Sanlin Community Health Service Center in 2015-2017, conducted a stroke screening test for the elderly population aged 65 and over in Sanlin area. The screening results are summarized and analyzed as follows.

\section{Materials And Methods}

\subsection{Research subject and Methods}

Sanlin Town is located in the southwest of Pudong New Area, with a registered population of 118,000 and 6 communities. This study conducted a screening of high-risk stroke patients in the elderly population in the Sanlin community. For the period from June 2015 to June 2017, the resident population $\geq 65$ years in Sanlin Community were screened for risk factors of stroke. Hospital outpatient and inpatient were excluded. Participants were fully informed and then signed the project informed consent form. A cross-sectional survey of 10,943 permanent residents 
of the community $\geq 65$ years old was conducted by cluster sampling. A face-to-face inquiry survey and related physical examinations were conducted by trained physicians with a standardized questionnaire and standardized survey methods. We collected basic information, family history, past medical history, medication record, smoking history, drinking history, exercise. Physical examination included height, weight, body mass index (BMI), blood pressure, pulse rate, and heart auscultation. Laboratory tests included blood lipids (TG, TC, HDL-C, LDL-C), fasting blood glucose, glycosylated hemoglobin, homocysteine, and electrocardiogram.

\subsection{Diagnostic criteria}

Cerebral stroke is defined as cerebral hemorrhage and cerebral infarction. It refers to the diagnosis of patients with neurologists based on relevant symptoms, signs and clinical examinations. Transient ischemic attack refers to unclear speech, hemiplegia, partial sensory disturbance, monocular black sputum, dizziness, unstable walking, etc., but MRI does not show any signs of cerebral infarction. The definition of hypertension refers to the Guidelines for the Prevention and Treatment of Hypertension in China (2010 Revision): systolic blood pressure $\geq 140 \mathrm{mmHg}$ and / or diastolic blood pressure $\geq 90 \mathrm{mmHg}$ without antihypertensive drugs or has been diagnosed with hypertension in the hospital. A history of diabetes, dyslipidemia, and atrial fibrillation are provided by patients with a basis for a definitive diagnosis by a community-level or higher hospital. Patients who smoke more than one cigarette per day for more than one year are regarded as smokers. Overweight refers to BMI $\geq 26 \mathrm{~kg} / \mathrm{m}^{2}$. The standard of physical exercise is $\geq 3$ times per week, $\geq 30$ minutes each time, and lasts for more than 1 year. Those who are engaged in moderate to severe physical labor are considered to have regular physical exercise.

The definition of high-risk stroke patients is a history of transient ischemic attack or history of stroke, or risk factors for stroke $\geq 3$.

\subsection{Quality Control}

Shanghai is one of the best cities for community health services in China. Its community health service center has established the regulation of family doctor service, which can effectively mobilize and follow up residents to ensure the quality of screening and follow-up work. The government and the community health centers carried out the publicity and education of the residents and the on-site organization work to ensure the screening work. The Shanghai Municipal Health Planning Commission has developed a screening work manual and work plan. As one of the stroke centers, our hospital is responsible for community doctor training, on-site guidance, and results review. Community hospital doctors in the department of preventive medicine are responsible for screening and registration of high-risk populations. Family doctors in community hospitals and health service stations are responsible for the follow-up.

\subsection{Statistical Analysis}

The data was analyzed using SPSS 22.0 software. The description of metrological data is expressed in mean \pm standard deviation. The measurement data description is represented by the count data description is expressed as a percentage. The measurement data were compared by $t$ test and the rate was compared by chi-square test. $\mathrm{P}<0.05$ is considered statistically significant.

\section{Results}

\subsection{Demographic information}


At the start point of the screening work in 2015 , there were 10,943 people $\geq 65$ years old in the Sanlin community. From 2015 to 2017, the number of screenings was 9,195 and the number of high-risk groups was 1,504. The proportion of high-risk groups was $16.78 \%$. Among them, there were 535 people with a history of transient ischemic attack or stroke. The detection rate was $5.82 \% .969$ people in high- risk group have $\geq 3$ risk factors. The detection rate was $10.54 \%$. The age of screened residents was $72.91 \pm 6.68$ years old. The detection rate of male high-risk population was $18.08 \%$ (739/4073) and $14.94 \%$ (765/5122) for female.

\subsection{Risk factors exposure}

The exposure rate of stroke risk factors in high-risk population was hypertension (83.71\%), overweight (53.86\%), hyperlipidemia (42.82\%), diabetes (39.83\%), and stroke history (35.57\%), smoking history (22.27\%), family history (20.21\%), lack of exercise (18.02\%) and atrial fibrillation (7.05\%). (Table 1)

Table 1

Exposure to risk factors for stroke in highrisk groups

\begin{tabular}{|ll|}
\hline Risk factors exposed & Percentage \\
\hline hypertension 1259 & 83.71 \\
\hline diabetes 599 & 39.83 \\
\hline hyperlipemia 644 & 42.82 \\
\hline atrial fibrillation 106 & 7.05 \\
\hline smoking history 335 & 22.27 \\
\hline overweight 810 & 53.86 \\
\hline Lack of exercise 271 & 18.02 \\
\hline Stroke history 535 & 35.57 \\
\hline Family history 304 & 20.21 \\
\hline
\end{tabular}

\subsection{Distribution of risk factors among high-risk groups of different genders}

Among the 969 high-risk groups with $\geqq 3$ risk factors the high-risk population of women with diabetes and hyperlipidemia was higher than men $\left(X^{2}=10.815, P=0.001 ; X^{2}=43.411, P<0.001\right)$. The rate of smoking and overweight of high-risk population in male were higher than women $\left(X^{2}=303.821, P<0.001\right)$. (Table 2) 
Table 2

Exposure of risk factors for high-risk groups of different genders

\begin{tabular}{|lllllllll|}
\hline Risk factors & Male & & Female & \multicolumn{3}{c|}{ Summary } & $\mathbf{X}^{2}$ & P \\
\hline & exposed & Percentage & exposed & Percentage & exposed & Percentage & & \\
\hline hypertension & 439 & 87.1 & 413 & 88.8 & 852 & 87.9 & 0.518 & 0.472 \\
\hline diabetes & 207 & 41.1 & 240 & 51.6 & 447 & 46.1 & 10.395 & 0.001 \\
\hline hyperlipemia & 212 & 42.1 & 294 & 63.2 & 506 & 52.2 & 42.567 & $<$ \\
\hline $\begin{array}{l}\text { Atrial } \\
\text { fibrillation }\end{array}$ & 36 & 7.1 & 31 & 6.7 & 67 & 6.9 & 0.027 & 0.869 \\
$\begin{array}{l}\text { smoking } \\
\text { history }\end{array}$ & 258 & 51.2 & 6 & 1.3 & 264 & 27.2 & 301.31 & $<$ \\
\hline $\begin{array}{l}\text { overweight } \\
\text { Lack }\end{array}$ & 318 & 63.1 & 330 & 50.9 & 648 & 66.9 & 6.416 & 0.011 \\
\hline exercise & 100 & 19.8 & 105 & 22.6 & 205 & 21.2 & 0.930 & 0.335 \\
\hline $\begin{array}{l}\text { Family } \\
\text { history }\end{array}$ & 149 & 29.6 & 159 & 34.2 & 308 & 31.8 & 2.1825 & 0.140 \\
\hline
\end{tabular}

\subsection{Hcy test results}

We define plasma Hcy >15 mmol/I as Hyperhomocysteinemia. Among the community members who participated in the screening, the detection rate of hyperhomocysteinemia was $53.72 \%$. The male detection rate was $63.8 \%$ and the female was $45.7 \%$. The difference was statistically significant. There was no significant difference in the detection rate of high homocysteinemia between high-risk groups and non-high-risk groups. (Table 3)

Table 3

Comparison of detection rates of hyperhomocysteinemia in different populations

\begin{tabular}{|llll|}
\hline group & non-hyperhcyemia(\%) & hyperhcyemia(\%) & P \\
\hline High-risk groups & $833(55.4)$ & $671(44.6)$ & \\
\hline Non-at-risk population & $3976(51.7)$ & $3715(48.3)$ & 0.385 \\
\hline Male & $1474(36.2)$ & $2599(63.8)$ & \\
\hline Female & $2781(54.3)$ & $2341(45.7)$ & $<0.01$ \\
\hline
\end{tabular}

\section{Discussion}

China attaches great importance to the prevention and control of cerebrovascular diseases. In 2011, the "Stroke Screening and Prevention Engineering Committee of the Ministry of Health" was set up by the former Ministry of Health to initiate the work of stroke screening and intervention to screen the high-risk population of stroke among people over 40 years old. In recent years, many screening results have been published. The results of screening in different areas and populations are not entirely consistent. Age is a risk factor that can not be intervened of stroke, especially in the population over 65 years old, the incidence of stroke increased significantly. This study screened the 
high-risk factors of stroke among community residents over 65 years old and studied the exposure of high-risk factors in this age group in Shanghai so as to provide evidence for the formulation of targeted prevention and control measures for the elderly.

Shanghai has established a relatively mature three-level prevention system for chronic diseases such as hypertension and stroke through the regulation of family doctor service. The screening results showed that the detection rate of high-risk population in Sanlin community was $16.36 \%$ which was similar to that reported by Fu Jianhui [3] in the Zhuqiao community in southern Shanghai. However, Fu Jianhui's screening target is community residents over 45 years old but the people we screened are residents over 65 years old. The detection rate of Fu's report is not lower than our detection rate, which may be related to the age reduction of risk factors such as hypertension, diabetes, hyperlipidemia and so on. The prevalence of hypertension, diabetes mellitus and hyperlipidemia did not decrease in the younger age group, so the detection rate of the high-risk group was not lower than that of older people. The results of screening in Hohhot showed that the detection rate of high-risk population over 60 years old was $26.15 \%[4]$, which was higher than that in Shanghai. It was consistent with the geographical distribution of stroke incidence in the southern part and northern part of China, suggesting that the distribution of high-risk population of stroke was also geographically different.

In our study, the exposure rate of risk factors were hypertension, overweight, hyperlipidemia, diabetes, stroke history, smoking history, family history, lack of exercise, and atrial fibrillation, decreasing accordingly. In Fu's study [3], hypertension and overweight were also the two most risk factors for exposure. Others were physical exercise, smoking, diabetes, dyslipidemia, family history of stroke, and atrial fibrillation [3]. It is suggested that the exposure rate of risk factors varies among high-risk groups of different ages. This phenomenon was also observed in a survey of a city in Sichuan Province [5]. The top three risk factors for high-risk strokes in the 70-84 years old group and the 85-year-old age group were hypertension, hyperlipidemia, and overweight, similar to our study. In the 40-54 age group and 55-69 years old patients with high risk of stroke, the top three risk factors are hypertension, smoking, and high blood lipids. It is important to perform public health education for this age group, to rationally adjust the dietary structure, and to promote a healthy lifestyle (smoking and alcohol cessation). Atrial fibrillation was the lowest risk factor in the study. During follow-up, $25.56 \%$ of the high-risk groups with cerebral infarction had atrial fibrillation, and most of them did not know that there was atrial fibrillation before the stroke. It suggests that the detection rate of atrial fibrillation is low and screening of atrial fibrillation should be strengthened.

We analyzed the exposure of risk factors for different gender. We found that among the high-risk groups with risk factors $\geq 3$, the smoking and overweight rate of male was higher than that of female. Women with diabetes and dyslipidemia of high-risk group were higher than that of male, which was consistent with Fu Jianhui's research. Smoking can cause vasospasm, increase blood pressure and accelerate arteriosclerosis, leading to cardiovascular and cerebrovascular events [6]. Abdominal obesity is an important factor in intracranial artery stenosis [7]. So it is necessary to emphasize a healthy lifestyle such as reasonable diet, weight control, smoking cessation and alcohol restriction for male high-risk groups. For women at high risk, to regular monitoring of blood sugar, blood lipid management and drug compliance should be strengthened.

Hyperhomocysteinemia (HHcy) is an important risk factor for cardiovascular and cerebrovascular diseases. Studies have shown that for every $1 \mu \mathrm{mmol} / \mathrm{l}$ increased plasma homocysteine, the risk of coronary atherosclerotic heart disease increases 1.6 times and the risk of cerebral vascular disease increases 1.8 times [8]. However, the prevalence of $\mathrm{HHcy}$ in the elderly population especially in high-risk stroke patients, is still unclear. Our study examined the blood Hcy value of the screening population and analyzed the distribution of HHcy in the high-risk population of stroke. Our results showed that the detection rate of hyperhomocysteinemia in the elderly over 65 years old is $53.72 \%$ and the 
detection rate of high-risk population is $44.6 \%$. A survey in Xi'an showed that $53.84 \%$ of the high-risk population of stroke had HHcy. Besides, the prevalence of HHcy was higher in people over 60 years old than those under 60 years old [9]. Univariate and multivariate analysis found that age was the most important risk factor for HHcy. Our study also found that the detection rate of hyperhomocysteinemia in men was higher than that in women, which was consistent with the study by Shang [10]. Our study did not find that the proportion of Hhcy in high-risk stroke patients was higher than that in non-high-risk groups.

Our study results show that the detection rate of high-risk populations in the Sanlin area is similar to that in other parts of Shanghai. Hypertension, overweight, and hyperlipidemia are the top three risk factors for exposure. For male population, the intervention of lifestyle such as weight control, smoking cessation and alcohol restriction should be emphasized, and the management of blood sugar and lipid should be strengthened in female population. Hyperhomocysteinemia has a high detection rate in the elderly and male population. It is necessary to pay attention to the management of Hcy in this group and timely supplement of folic acid. On the basis of the above, regular follow-up management should be carried out in combination with the occurrence of risk factors in order to achieve the goal of preventing stroke.

\section{Declarations}

\section{Disclosure statement}

None declared. All authors have seen and approved the manuscript and consent for publication. No on reported any conflict of interests.

\section{Ethics}

This study is approved by ethics committee of East Hospital, Tongji University School of Medicine,Shanghai, China.

\section{Author Contributions Statement}

LYL performed the statistical analysis and drafted the manuscript. $L F, X Y, F F L, H D$ and ZL collected the data. YMY double-checked the statistical analysis. YMY and BZ designed the study, supervised the study, double-checked the statistical analysis and revised the manuscript.

\section{Data availability statement}

Data will be available upon request to corresponding authors.

\section{Acknowledgements}

This study was funded by the National Natural Science Foundation of China [grant numbers 81701039]; Young Excellent Talents in Tongji University (22120180604), and Scientific Research Projects of Shanghai Health and Family Planning Commission [Grant No. 201640388]. We thank all participants in this study.

\section{References}

1 Zhang Runhua, Liu Gaifen, Pan Yuesong, et al. Epidemiology of Stroke in the World. Chinese Journal of Stroke[J] $.2014,9(9): 767-773$ 
2 Wang Yude. Strategies and Tactics for Prevention and Control of Stroke in China[J]. Journal of Medical Research, 2012, 41(2): 1- 2.

3 Gao Lili, Fu Jianhui, Wang Weizhong, et al. A report on a screening of high-risk population for stroke in a community of Pudong New Area, Shanghai. Fudan University Journal of Medical Sciences[J].2017, 44(1):82-86.

4 Zhu Run-xiu, Yuan Jun, Chang Hong,etal. Research of screening results in high-risk population of stroke in Hohhot. Chinese Journal of Evidence-Bases Cardiovascular Medicine[J].2016,8(12):1455-1457

5 Wang Li, Chen Jinhua, Zhao Yuzhang,et al. Screening and analysis of risk factors for stroke high risk population in hospitalized patients. Medical Journal of West China[J]. 2017, 29(6):850-853.

$6 \mathrm{Li} \mathrm{Tao,} \mathrm{Zhang} \mathrm{Yunling,} \mathrm{Zhao} \mathrm{Hui,et} \mathrm{al.} \mathrm{Study} \mathrm{on} \mathrm{the} \mathrm{Correlativity} \mathrm{between} \mathrm{Smoking} \mathrm{and} \mathrm{Carotid} \mathrm{Atherosclerosis} \mathrm{for}$ High Risk Group of Stroke. Chinese Journal of Integrative Medicine on Cardio/Cerebrovascular Disease[J].2015,13(2): 175-178.

7 Zhang Xu,Chang Ying , Liu Songyan. Study on the correlation between metabolic syndrome and intracranial and extracranial artery stenosis in patients with ischemic cerebrovascular disease.Chinese Journal of Laboratory Diagnosis[J]. 2017, 21(12): 2148-2150

$8 \mathrm{He} \mathrm{Y,Li} \mathrm{Y,Chen} \mathrm{Y,etal.} \mathrm{Homocysteine} \mathrm{level} \mathrm{and} \mathrm{risk} \mathrm{of} \mathrm{different} \mathrm{stroke} \mathrm{types:a} \mathrm{meta-analysis} \mathrm{of} \mathrm{prospective}$ observational studies.Nutr Metab Cardiovasc Dis[J].2014,24:1168-1165.

9 Ma Hongni,Yan Fei,Li Zaili,etal. Distribution of hyperhomocysteinemia in community population at high risk of ischemc stroke in Xi'an. Chinese Journal of Geriatric Heart Brain and Vessel Diseases[J].2015,17(8): 830-833.

10 SHANG Lu, SUN Fenyong. Levels of serum homocysteine in middle-aged and elderly people in Dongjing community of Shanghai and their influence factors. Laboratory Medicine[J]. 2017, 32(9): 760-764. 